\title{
Ein erstes sicheres Vorkommen von periglazialem Steinnetzboden im Norddeutschen Flachland
}

\author{
von Heinrich Wortmann, Krefeld
}

Mit 3 Abbildungen im Text

Zusammenfassung. Bei Stadtlohn im westlichen Münsterland/Westfalen wird ein erstes sicheres weichselperiglaziales, sohlenständiges Steinnetzwerk aus dem lehmigen Abraum eines Turon-Kalksteinbruches beschrieben. Durch etagenförmig angelegte Horizontal- und Vertikalschnitte ist eine etwa $2000 \mathrm{qm}$ große Fläche aufgeschlossen, die einen guten Überblick über die räumliche Erscheinung bietet. Neben unregelmäßigem Steinnetzwerk und vereinzelten Steinstreifen sind auch wohlgeformte Steinpolygone vorhanden. Aufbau der Steinrahmen, Körnung und stoffliche Zusammensetzung der Feinerdebeete, in denen warmzeitlicher „Kalksteinbraunlehm" und Saale-Grundmoräne aufgearbeitet sind, werden erläutert. Eine genetische Deutung wird versucht und auf die Ähnlichkeit der Formen bei anderen periglazialen Frostböden hingewiesen.

A bst ract. Near Stadtlohn in Western Westphalia (North Germany) a well developped "patterned ground" (stone polygons, stone strips etc.) is described. It has been found in the loamy overburden of a limestone quarry. The loamy material consists of a loamy brown limestone soil and of boulder clay of the Saale-Glaciation. The age of this patterned ground can be determined as Weichsel-Periglacial. The material of the stone-borders comes from the underlaying limestone. The formation and mechanism are discussed and relations to other periglacial soils with frost structures are mentioned.

Periglaziale Frostbodenerscheinungen, die im Norddeutschen Flachland zumeist nur unter einer schützenden Decke jüngerer Ablagerungen gut erhalten geblieben sind, kommen als Eiskeile, Taschen-, Tropfen- und Würgeböden in solcher Häufung und an so vielen Orten vor, daß neue Vorkommen nur noch der regionalen Vollständigkeit halber registriert werden. Es stehen genügend, z.T. vorzügliche Abbildungen für die Erscheinungen zur Verfügung.

Strukturböden im Sinne der engen Fassung von Meinardus (1912, 1929), der darunter nur frostbedingte Böden aus unhomogenem Material mit deutlicher Detritussortierung zu Streifen, Ringen, Netzen u. ä. versteht, sind im Norddeutschen Flachland und auch in Holland (van der VleRK \& FlorschüTz 1949) nicht bekannt. STEEgER (1944, S. 530), der sich um die Erforschung der periglazialen Bodenformen im Niederrheingebiet besondere Verdienste erworben hat, erwähnt ein Vorkommen von zwei auffälligen Kreisen dichter Blockbestreuung von etwa $10 \mathrm{~m}$ Durchmesser, die nach Abtragung einer deckenden Sandschicht in der Eltener Stauchmoräne freigelegt waren, als möglicherweise echten Strukturboden; jedoch betont er in einer mündlichen Mitteilung (1956), daß sich im Niederrheingebiet Strukturböden in Form von Steinnetzwerk nicht haben nachweisen lassen.

Gesicherte Vorkommen von fossilen Strukturböden aus den deutschen Mittelgebirgen sind schon länger bekannt (Dücker 1937, Gellert 1933, Poser \& Hövermann 1951 Hempel 1955). Diese Vorkommen waren aber zumeist nicht gut aufgeschlossen. Abbildungen, die Aufsicht und senkrechten Schnitt zusammen bringen, sind selten, was bei der großen Schwierigkeit einer Aufgrabung von Strukturböden verständlich ist. Häufig dagegen sind gute Aufsichtsbilder von rezenten Strukturböden aus den Frostklimaten der Polargebiete als auch der subnivalen Stufe der Hochgebirge (u. a. Poser 1931, Troll 1944).

Auf der Tagung der DEUQUA (August 1955 in Laufen bei Saizburg) und auf der Tagung der Deutschen Bodenkundlichen Gesellschaft in Göttingen (September 1955) 
wurden vom Verfasser kurze Mitteilungen gemacht, daß jetzt auch im nordwestdeutschen Flachland ein periglazialer Steinnetzboden gefunden wurde ${ }^{1}$ ).

Im westlichen Münsterland gibt es bei Stadtlohn-Hundewick, Oeding, Wessum-Graes usw. eine Reihe niedriger Höhenrücken, deren Kern aus flacheinfallenden Kalksteinen der Oberen Kreide besteht. BÄRTLING (1913) und BENTZ \& GöRz (1933) erwähnen aus diesem Gebiet auffällige Gebilde von fettem braunem Lehm mit vereinzelten Kalksteinbrocken, die zapfenförmig in die unverwitterten Kalksteine eingreifen. Sie werden von den $\mathrm{Ge}-$ nannten als „Geologische Orgeln“ bezeichnet, die lediglich durch die Lösungstätigkeit des Wassers im Kalkstein entstanden sind, also kleine Dolinen darstellen. BäRTLING betont ausdrücklich, daß es sich nicht um Gletschertöpfe oder Gletschermühlen handelt, doch weist er darauf hin, daß möglicherweise auch diluviales Material in den „Geologischen Orgeln" enthalten ist. Derartige Gebilde wurden 1933 in dem gleichen Aufschluß beschrieben, in dem jetzt der Strukturboden gefunden wurde. Sie stellen den Rest eines warmzeitlichen (tertiären oder interglazialen) Verwitterungsbodens dar, einen Kalksteinbraunlehm im Sinne der Definition von Kubiena (1948, S. $80 \mathrm{ff}$.). Dafür spricht die von Bentz \& Görz (1933, S. 48/49) angeführte Analyse, insbesondere der hohe Anteil an löslicher Kieselsäure sowie der plastische, klebrige Charakter der Bodenart und die rotbraune Farbe.

Genetisch haben diese als "Geologische Orgeln" bezeichneten Formen mit den Strukturböden nichts zu tun; lediglich hat der Kalksteinbraunlehm Material für die Feinerde des Steinnetzbodens geliefert. In allen Fällin jedoch, in denen diluviales Material vorkommt (B̈̈RTLING 1913), muß eine Strukturbodenbildung durch Einwirkung der diluvialen Regelation vorliegen.

Im Steinbruch der Westfälischen Kalkwerke in Stadtlohn-Hundewick unmittelbar westlich der Landstraße von Stadtlohn nach Südlohn im westlichen Münsterland (Messtischblatt Stadtlohn Nr. 4007) war im Frühjahr 1955 durch Abraumarbeiten ein gut aus-
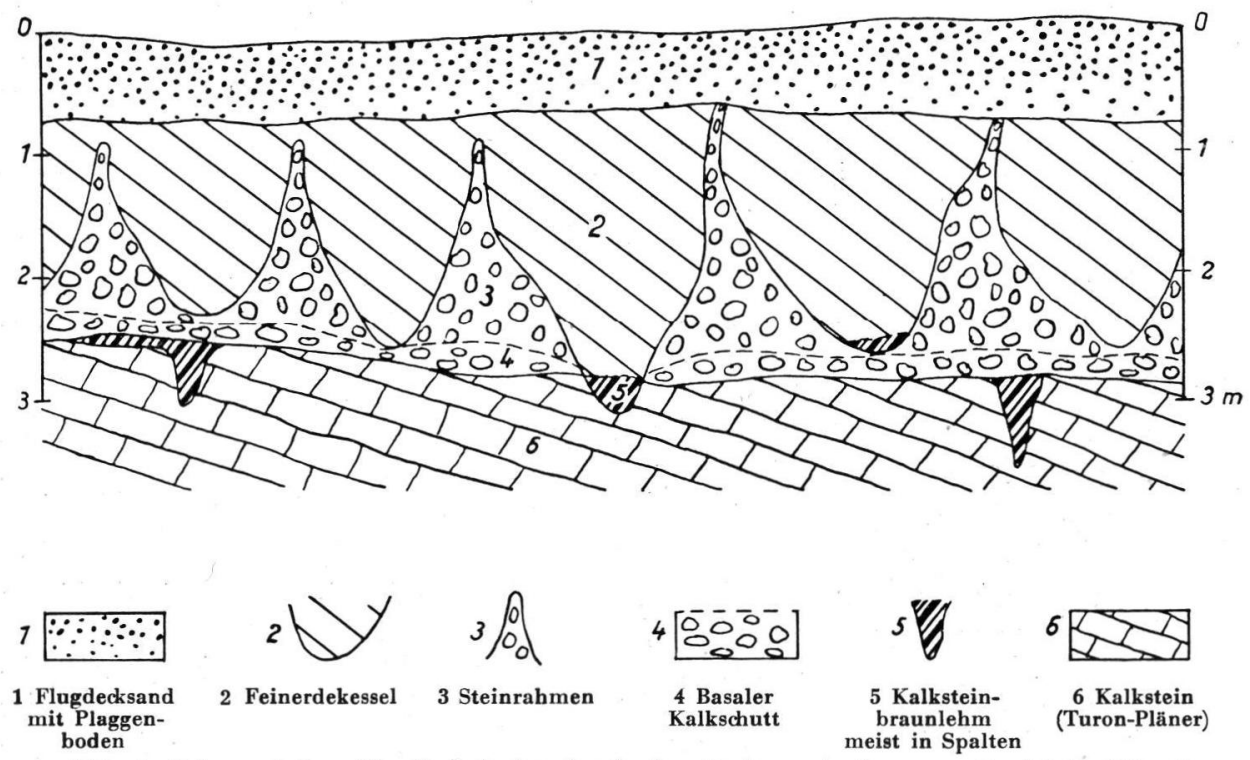

Abb.1. Schematischer Vertikalschnitt durch den Steinnetzboden von Stadtlohn/Westf.

1) In der Zwischenzeit ist von ScHeNk (1955) ein senkrechter Schnitt eines fossilen Strukturbodens bei Tschenstochau unweit des Warthetales veröffentlicht worden, ebenfalls brachte HEMPEL (1955) einen schematischen Schnitt durch ein Strukturbodenvorkommen im Muschelkalk bei Göttingen. 
geprägter Stukturboden aufgeschlossen. Die abgedeckte Fläche war $15 \mathrm{~m}$ breit und $120 \mathrm{~m}$ lang. Das Liegende in der Kalksteingrube bilden die in etwa $10 \mathrm{~m}$ Mächtigkeit aufgeschlossenen, mit $12^{\circ}$ nach OSO einfallenden Plänerkalke des Turons. Während an der Abbausohle noch Rotpläner angetroffen wurde, besteht der obere Teil aus knollig zerfallenden Kalken der Labiatus- und Lamarckizone. Darüber folgt der im Strukturboden aufgearbeitete Geschiebelehm der Saalevereisung, überdeckt von spätpleistozänen Flugdecksanden in einer Mächtigkeit von $10-80 \mathrm{~cm}$, die eine Plaggenauflage von etwa $60 \mathrm{~cm}$ tragen (Abb. 1).

Die Landstraße an der Ostseite des Steinbruchs verläuft auf einem schwachen Höhenrücken in $60 \mathrm{~m}$ ü. NN, von dem aus das Gelände mit geringer Neigung von 2-30 nach $\mathrm{O}$ und W abfällt. Die flachgewellte Oberfläche des ausstreichenden Kalksteins ist fast horizontal oder leicht nach $\mathrm{O}$ geneigt, da die Mächtigkeit der eiszeitlichen Deckschichten nach O zunimmt.

Nach Abtrag des Plaggenbodens und der Flugdecksandschichten zeigen sich auf einem großen Teil der Abraumfläche, besonders in den leichten Mulden, schöne, mehr oder weniger regelmäßige, rundliche, längliche, z.T. aber auch polygonal geformte Steinkränze aus hellgrauen Kalksteinknollen, die Feinerdefelder aus dunkelbraunem Lehm umschließen. Die Steinkränze sind 3-5 cm breit, die Kalkknollen etwa walnussgroß, die Feinerdefelder von wechselnder Größe zwischen 0,5-2,5 m. Senkrecht gelegte Schnitte, die durch einen weiteren Etagenabbau modellhaft freigelegt wurden, ließen erkennen, daß die in der Aufsicht schmalen Steinkränze nur der oberste Teil von sich nach unten verdickenden Steinmauern sind, die mit sehr breiter Basis dem unverwitterten Kalkgestein aufsitzen.

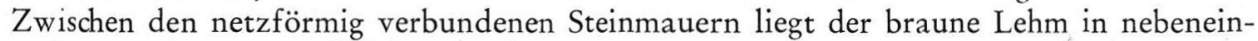
ander geordneten rundlichen Kesseln eingebettet. Wir haben also ein typisches sohlenständiges Steinnetzwerk (nach der Definition von Poser 1931) vor uns. Einen Aufblick auf die Abraumfläche von der Höhe des Baggerarmes aus gibt Abb. 2.

Die Größe der Kalksteinknollen in den Kesselwänden nimmt im allgemeinen von oben nach unten zu. Während oben in den dünn ausgezogenen Schwänzen (Abb. 2) Steine von Walnußgröße vorherrschen, sind sie im unteren Teil durchschnittlich ei- bis faustgroß. Offenbar bilden sich bei der Verwitterung keine größeren Knollen, oder sie zerfallen schnell bis zu mittleren Größen, da auch in der unterlagernden 10-20 cm mächtigen Kalkschuttzone keine größeren Knollen gefunden wurden. Besonders bemerkenswert ist die sperrige Lagerung der Knollen in den Steinmauern, ohne Ausfüllung der Hohlräume durch Lehm. Nur im oberen Teil, in der Kontaktzone zwischen Strukturboden und Flugdecksand, sind die Zwischenräume mit einem wahrscheinlich später eingeschwemmten, durch Vermischung von Lehm und Flugdecksand entstandenen lehmigen Sand bis zu etwa $20 \mathrm{~cm}$ Tiefe ausgefüllt.

Der Feinerdekesseldurchmesser nimmt nach unten hin immer mehr ab und beträgt auf der zweiten Abbauterrasse in etwa $80 \mathrm{~cm}$ Tiefe unter der Decksandauflage (im weiteren mit u. D. bezeichnet) noch zwischen 40 bis $70 \mathrm{~cm}$. Eine Aufsicht auf diesen Horizontalschnitt gibt Abb. 3, auf der auch deutlich der polygonale Querschnitt eines Feinerdebeetes zu erkennen ist. Der Kesselboden der Feinerde liegt ziemlich regelmäßig bei $1,30-1,50 \mathrm{~m}$ Tiefe u. D. und folgt kleineren Wellungen der ehemaligen Oberfläche.

Der braune Lehm ist sehr gleichmäßig beschaffen und hat nur einen sehr geringen Anteil von ganz unregelmäßig verteilten Steinen. Neben Kalksteinknöllchen, zerbrochenen und ganzen Toneisensteingeoden sowie anderen Gesteinsbröckchen aus der näheren Umgebung findet sich auch nordisches Material verschiedener Größe. Zwei größere nordische Blöcke von $20-35 \mathrm{~cm}$ Länge waren in dem Strukturbodenmaterial enthalten, doch konnte ihre genaue ursprüngliche Lage nicht mehr festgestellt werden. Gelegentlich ist im unteren Teil der Kessel der Lehm rötlicher und plastischer, wobei es sich um einen höheren Anteil von Kalksteinbraunlehm an der Feinerde handelt. Einige Kessel sind ringsherum an der 


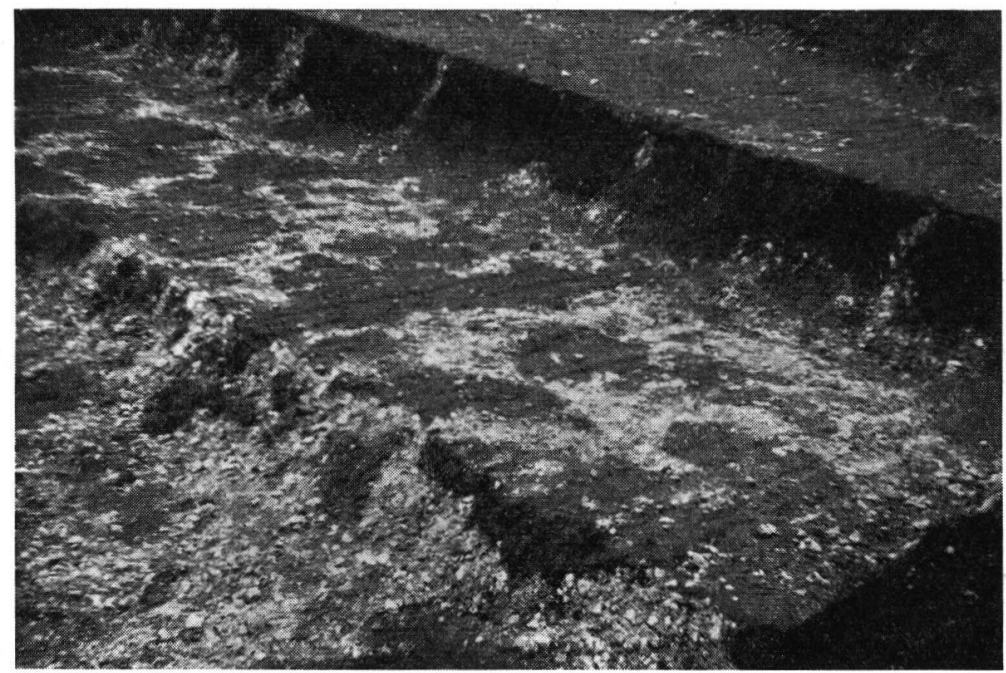

Abb. 2 Steinnetzboden von Stadtlohn/Westfalen

Schräge Aufsicht auf das durch 3 Horizontal- und 3 Vertikalschnitte freigelegte Steinnetzfeld. Der untere Horizontalabschnitt (im Bild links unten) liegt in der Kalksteinschuttzone über dem festen Kalkstein und hat nur einzelne, noch tiefer reichende Lehmflecken. Der darüber folgende Vertikalschnitt zeigt die dunkle Lehmfüllung zwischen breiten Kalksteinmauern. Der mittlere Horizontalschnitt zeigt das Nebeneinander von breiteren, hellen Steinnetzen und dunklen Lehmkesseln. Im Hintergrund, beim Übergang in den nächsten Vertikalschnitt sind die Steinwände schon bedeutend schmaler und die Lehmfelder entsprechend breiter. Der obere Horizontalschnitt zeigt nur noch perlschnurartige Steinnetze, die im obersten Vertikalschnitt (im Bild ganz rechts oben) auslaufen.

Foto Wortmann, Februar 1955

Grenze zu den Steinmauern mit einem dunkelbraunen bis braunschwarzen tonigen Lehm bis lehmigen Ton von etwa 10-20 cm Mächtigkeit überkleidet. Dieser dunkle Ton findet sich auch außerhalb der Strukturbodenbildung auf der Kalksteinoberfläche. Seine Bedeutung bei der Genese des Strukturbodens ist noch unklar und bedarf weiterer Untersuchung.

Die Korngrößenuntersuchung, die ich Herrn Dr. WERner (Amt für Bodenforschung, Krefeld) verdanke, zeigt den Unterschied der Korngrößenzusammensetzung im unteren, leicht mit Flugdecksand vermischten Teil des Plaggenbodens und der Feinerde eines Strukturbodenkessels aus drei verschiedenen Tiefen. Es ist besonders zu bemerken, daß die oberste Probe der Kesselfeinerde direkt über einem der auslaufenden Steinschwänze, etwa $10-20 \mathrm{~cm}$ unter der Flugsanddecke, entnommen wurde (Tab. 1).

$$
\text { T a be } 11 \text { e } 1
$$

Korngrößenverteilung im Bodenprofil des Steinnetzvorkommens bei $\mathrm{Stadtlohn}$

\begin{tabular}{|c|c|c|c|c|c|c|c|}
\hline & & \multicolumn{6}{|c|}{ Korngröße in $\mathrm{mm}$ (in $\% \mathrm{~d}$. Feinbod. $<2 \mathrm{~mm}$ ) } \\
\hline $\begin{array}{l}\text { Tiefe u. } \mathrm{O} . \\
\text { in } \mathrm{cm}\end{array}$ & Probe & $<0,002$ & $\begin{array}{r}0,002 \\
-0,005\end{array}$ & $\begin{array}{r}0,005 \\
-0,02\end{array}$ & $\begin{array}{r}0,02 \\
-0,05\end{array}$ & $\begin{aligned} & 0,05 \\
- & 0,2\end{aligned}$ & $\begin{array}{r}0,2 \\
-2,0\end{array}$ \\
\hline & $\begin{array}{l}\text { Plaggenboden } \\
\text { aus Flugdecksand }\end{array}$ & 6,5 & 1,0 & 4,8 & 12,0 & 18,0 & 57 \\
\hline-100 & $\begin{array}{l}\text { Feinerde oben unter dem } \\
\text { Flugdecksand }\end{array}$ & 24,1 & 1,1 & 8,2 & 9,5 & 33,0 & 24 \\
\hline 180 & $\begin{array}{l}\text { Feinerde aus der Mitte } \\
\text { des Kessels }\end{array}$ & 17,9 & 4,4 & 6,9 & 5,0 & 37,2 & \\
\hline 230 & Feinerde an der Grenze & 22,3 & 1,3 & 6,9 & 9,4 & 33,3 & \\
\hline
\end{tabular}

Die von Dücker (1937) festgestellte Häufung der Lößfraktion in der Feinerde von Strukturböden ist hier also nicht vorhanden und kann auch nicht erwartet werden, $\mathrm{da}$ 
Geschiebelehm und Kalksteinbraunlehm, also bereits aufgearbeitete Bodenarten, das Material der Feinerde lieferten. Der gröbere Anteil von $0,05-2,0 \mathrm{~mm}$, der mit rund $57 \%$ sehr groß ist, ist durch die Frostvorgänge nicht zur Lößfraktion aufgearbeitet worden.

Eine Untersuchung des Schwermineralbestandes der Feinsandfraktion $(0,1-0,2 \mathrm{~mm})$ ergab für die Feinerde des Stadtlohner Steinnetzwerkes (nach H. WERnER):

$\begin{array}{lc}\text { Granat } & 45 \% \\ \text { Epidot } & 22 \% \\ \text { Hornblende } & 14 \% \\ \text { Staurolith } & 9 \%\end{array}$

Interessant ist der Vergleich des Schwermineralgehaltes des etwa $30 \mathrm{~km}$ östlich gelegenen kalkhaltigen, etwas streifigen Lösses bei Schapdetten im südöstlichen Teil der Baumberge, der in einer Mächtigkeit von mehreren Metern dem obersenonen Baumberge-Sandstein aufliegt. Die Probe ist aus dem C-Horizont in etwa $2 \mathrm{~m}$ Tiefe u. O. entnommen:

$\begin{array}{lc}\text { Granat } & 42 \% \\ \text { Epidot } & 18 \% \\ \text { Hornblende } & 18 \% \\ \text { Staurolith } & 9 \%\end{array}$

Die Kesselfeinerde von Stadtlohn und der Baumberger Löß zeigen den gleichen Schweremineralbestand, die prozentualen Unterschiede sind sehr gering. Da dieser Bestand aus dem anstehenden Kreidegestein nicht herzuleiten ist, entstammt er dem Antransport von Fremdmaterial durch die Saalevereisung.

$\mathrm{Zu}$ erwähnen ist noch das Vorkommen von Streifenboden an der Westseite der Stadtlohner Grube, wo ein lokal stärkerer Neigungswinkel der Kalksteinoberfläche die Deformierung des Steinnetzwerkes zu nebeneinanderliegenden, etwa $30-40 \mathrm{~cm}$ breiten Streifen von Feinerde und Steinmauern bewirkt hat. Die Steinmauern sind hier ebenfalls sohlenständig, da sie Kontakt mit dem unterlagernden festen Kalkstein haben.

Das Alter des Stadtlohner Steinnetzbodens ist weichsel-periglazial, da die eingearbeitete Grundmoräne saaleglazial und die überlagernde Flugsanddecke spätglazial ist. Über die

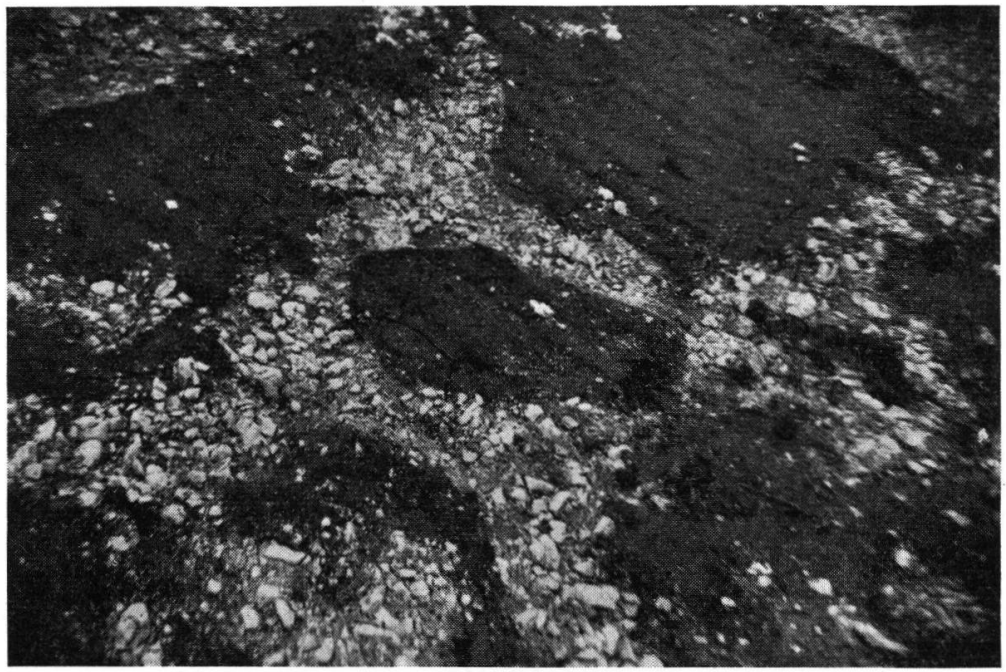

Abb. 3 Steinnetzboden von Stadtlohn/Westfalen

Steile Aufsicht auf den mittleren Horizontalschnitt von Abb. 2. In der Mitte unregelmäßiges Fünfeck von braunem Lehm (ca. $60 \times 30 \mathrm{~cm}$ ), eingerahmt von hellem Kalkschutt von 15 bis $30 \mathrm{~cm}$ Breite. Die Kalksteinbröckchen auf dem dunklen Lehm sind durch den Bagger verschleppt und entstammen den Steinrahmen. Foto WORTMANN, Februar 1955 
von den vorrückenden Eismassen des Saaleglazials stark abgehobelte Oberfläche der warmzeitlich (tertiär oder interglazial) verwitterten Kreide, wobei wahrscheinlich nur die tiefsten Teile des in Auslaugungsformen vorhandenen roten Kalksteinbraunlehms, geschützt durch randliche feste Gesteine, erhalten blieben, legte sich nach Abschmelzen des Eises die Grundmoräne. Sie enthielt hier nordisches und einheimisches Material in starker Vermischung und Aufbereitung. Ob im nachfolgenden Saale-Warthe-Interstadial die Strukturbildung schon begann, läßt sich nicht entscheiden. Die stärkste Strukturbildung muß jedoch im Weichselperiglazial stattgefunden haben, da sich eeminterglaziale Bodenbildungen nirgends finden lassen und das spätglaziale Alter des auflagernden Flugdecksandes gesichert ist.

Das Stadtlohner sohlenständige Steinnetzwerk weist beim Vergleich mit anderen in der Literatur beschriebenen einige bemerkenswerte Abweichungen auf (vergleiche hierzu u. a. Poser 1931, 1954, Dücker 1937, Troll 1944, Hempel 1955, Schenk 1955). Die Abweichungen liegen in folgendem:

1. Die Größensortierung der Steine innerhalb der Steinmauern ist hier so, daß oben (in den Schwänzen) die kleinsten, unten die größeren liegen.

2. Mit 1,5 m Tiefe u. D. ist das Stadtlohner Vorkommen mächtiger als alle bisher bekannt gemachten, die $0,8 \mathrm{~m}$ nicht überschreiten.

3. Das Stadtlohner sohlenständige Steinnetzwerk ist nicht isoliert, wie es für andere Vorkommen betont wird, sondern breitet sich in einem aneinanderschließenden Netz über eine größere Fläche aus. Auch Hempel's (1955) schematischer Schnitt durch den Strukturboden bei Göttingen gibt mehrere nebeneinanderliegenden Formen auf $11 \mathrm{~m}$ Breite an.

Die Größenabnahme der Steine nach oben deutet darauf hin, daß wir einen gealterten Steinnetzboden, eine Zerfallsform, vor uns haben. Die Alterung kann im gleichen weichselperiglazialen Zyklus wie die Entstehung des frischen erfolgt sein, wie die Beobachtungen über Entwicklungsreihen und Alterungsformen rezenter, benachbarter Strukturböden durch Högbom (1927) in Schweden, Nansen (1922) sowie Huxley \& Odell (1924) in Spitzbergen zeigen. Die genannten Autoren haben nebeneinander Übergangsformen vom vollausgebildeten Strukturboden bis zur völligen Auflösung der Steine zu Feinerde feststellen können. Dieser Alterungsprozeß hat offenbar auch im oberen Teil des Stadtlohner Strukturbodens an einigen Stellen zur völligen Verwitterung der Steine geführt. Die Untersuchung einer steinfreien Bodenprobe, die direkt über einem der auslaufenden Schwänze entnommen wurde, ergab ihre Zugehörigkeit zur Feinerde und nicht zum Flugdecksand (s. S. 122). Ein „Brodelpflaster“, das durch spätere Ausblasung (Dücker 1933) in frostbedingten Böden häufig entsteht, hat sich daher hier nicht gebildet. Die ebene Grenzfläche des Steinnetzbodens zur überlagernden Flugsanddecke weist ebenfalls darauf hin, daß die Strukturformen schon lange vor der Decksandüberlagerung inaktiv waren. Auf der etwas weiter westlich im Steinbruch gelegenen kleinen Kuppe (der Kreideoberfläche) muß entweder eine primär geringere Strukturbodenbildung vorhanden gewesen oder Ausblasungs- bzw. Abtragungsvorgänge stärker wirksam gewesen sein, da dort unter dem Flugdecksand nur noch Strukturbodenformen geringerer Mächtigkeit erhalten sind. Da aber auch hier keine Steinsohle vorhanden ist, ist anzunehmen, daß auch der Steinanteil oben im Strukturboden vor der Abtragung weitgehend verwittert war.

Die von POSER (1947 a und b) aus verschiedenen Frostbodenformen abgeleitete weichselperiglaziale Auftautiefe von ca. 2 Metern, die für seine glazial-maritime Übergangsprovinz in Nordwestdeutschland charakteristisch ist, stimmt gut mit der unteren Grenze des Stadtlohner Strukturbodenvorkommens überein, da die Tiefe unter Flugsanddecke als Mindestmaß angesehen werden muß.

Die Geschlossenheit des Strukturbodennetzes über eine größere Fläche weist auf reichliches Verwitterungsmaterial zu Beginn der Regelationsprozesse, Voraussetzungen, die im Eem-Interglazial durch Bodenbildungsprozesse geschaffen sein könnten. Offenbar 
herrschte zu den breiten flachen Mulden der Kalksteinoberfläche ein stärkerer Wasserzustrom, und die hierdurch gegebene günstige „hydrologische Position“ ist für die Größe und Aktivität der Steinnetze und ihren örtlichen Zusammenhang ein wesentlicher Faktor.

Es muß herausgestellt werden, daß das Stadtlohner Strukturbodenvorkommen zur Frage der Genetik und der Mechanik keine gute Handhabe bietet. Die Aufwärtswanderung der Steine im Feinerdeteil des Strukturbodens durch „Auffrieren“ und das seitliche Abgleiten im oberflächlich aufgetauten, noch gewölbten aktiven Frostboden durch Gravitationskräfte (POSER 1934, SCHENK 1955) kann hier wegen der Alterungsform nicht mehr erkannt werden. Eine Seitwärtsbewegung von unten her (HEMPEL 1955) ist wegen der ursprünglich knolligen Form der Kalksteine nicht zu belegen, da sich keine bestimmte Ausrichtung des Steinmaterials durch Einregelung festlegen läßt. Die gesamte Anordnung von Steinrahmen und Feinerdekesseln läßt allerdings kaum einen Zweifel darüber, daß die Steine sowohl aufwärts gewandert als auch seitlich verschoben sein müssen.

Eine weitere Verbreitung von vereinzelten Strukturböden im Münsterland ist bereits gelegentlich bei bodenkundlichen Kartierungsarbeiten vom Verfasser festgestellt. SW von Rheine treten ähnliche wannen- und zapfenförmige Gebilde an der Oberfläche der Cenoman-Kreide unter Plaggenboden auf, deren Untersuchung im Gange ist. Auch in den großen Aufschlüssen der Beckumer Zementindustrie in Obersenon-Kalksteinen, die mit Tonmergeln wechsellagern, zeigen sich vereinzelt deutlich einregulierte sohlenständige Kalkplattenrahmen zwischen Feinerdefeldern.

Es ist auffällig, daß die bisher im Münsterland bekanntgewordenen Steinnetzwerke alle auf Kalkstein vorkommen. Diese Bevorzugung des Kalksteins ist kein Zufall, sondern eine „edaphische Begünstigung “ des Strukturbodenphänomens im Sinne von Troll (1944, S. 592 f.), der darunter eine aklimatische Verstärkung versteht, da über einer Felsunterlage die Materialsortierung besonders scharf ausgeprägt ist. Einen weiteren Hinweis auf die Bevorzugung der Kalksteine geben die Untersuchungen (u. a. von DückER 1940) über die Abhängigkeit der Frostgefährdung eines Bodens von seiner Korngrößenzusammensetzung, aus denen ersichtlich ist, daß die Kolloidfraktion des Bodens ( $0,002 \mathrm{~mm}$ ) als wichtiger Faktor bei der Strukturbodenbildung angesehen werden muß, da sie den höchsten Zustrom von Wasser zur Bodenfrostfront aufzuweisen hat. In Kalksteinverwitterungsböden kommen Kolloidtone in der Regel reichlich vor.

$\mathrm{Da}$ in Nordwestdeutschland eine große Anzahl von anderen Frostbodenformen (Tropfen, Taschen, Eiskeile) gefunden sind, wäre es interessant, vergleichende Untersuchungen anzustellen, die die Genetik der verschiedenen Erscheinungsformen der Frostböden weiter klären könnten. Ein gutes Vergleichsobjekt zu dem Stadtlohner Strukturboden ist der periglaziale Taschenboden bei Haltern/Westfalen, der von StEusloff (1952) als frühsaaleeiszeitlich angesprochen wird. Senkrechte Schnitte dieses Bodens (STEusLoff 1952, S. 305, Abb. 1) zeigen der Form nach eine überraschende Ähnlichkeit mit entsprechenden Schnitten des Stadtlohner Strukturbodens. Die „Kesselwände“ des Halterner Taschenbodens entstammen dem dort anstehenden weißlichen, lockeren untersenonen Quarzsand, der Inhalt der Taschen besteht aus einem Gemisch von Sand, Beckentonen und Löß mit Eisenschwartenstückchen, wobei eine deutliche Sortierung der Steine zu konzentrischschalenförmigen Gebilden parallel zur Taschenaußenwand zu erkennen ist. Der Frage, ob sich aus dieser Ahnlichkeit der Formen genetische Hinweise ergeben, wird man erst nachgehen können, wenn bei Haltern große Horizontalschnitte wie bei Stadtlohn freigelegt und untersucht sind.

\section{Literaturverzeichnis}

Bentz, A. \& Görz G.: Erläuterungen zur Geologischen Karte Blatt Stadtlohn. - Lfg. 293, Nr. 2209, Berlin 1933

BÄRtLING, R.: Geologisches Wanderbuch f. d. niederrheinisch-westfälischen Industriebezirk. Stuttgart 1913 
Dücker, A.: Frosteinwirkung auf bindige Böden. - Straßenbau-Jahrb. 1939/40, Berlin 1940. - Über Strukturboden im Riesengebirge. Ein Beitrag zum Bodenfrost- und Lößproblem. Z. deutsch. geol. Ges. 89, 1937. - - Steinsohle oder Brodelpflaster. - Centralbl. f. Min. etc., Abt. B, 1933.

Hempel, L.: Messungen an eiszeitlichem Strukturboden auf dem Göttinger Muschelkalk. - Neues Jahrb. Geol. Paläont., Mh., Stuttgart 1955.

Нӧявом, В.: Beobachtungen aus Nordschweden über den Frost als geologischer Faktor. - Bull. Geol. Inst. Upsala 1927.

Huxlay, I. S. \& Odell, N. E.: Notes on surface markings in Spitsbergen. - Geogr. Journ. 63, 1924.

Kubiena, W. L.: Entwicklungslehre des Bodens. - Wien 1948.

Meinardus, W.: Verteilung der Böden an der Erdoberfläche und ihre Ausbildung. Arktische Böden. - Hdb. d. Bodenlehre, Bd. III, 1930.

NANSEn, Fr.: Spitzbergen. - Leipzig 1922.

Poser, H.: Beiträge zur Kenntnis der arktischen Bodenformen. - Geol. Rundschau 22, 1931. Dauerfrostboden und Temperaturverhältnisse während der Würmeiszeit im nicht vereisten Mittel- und Westeuropa. - Naturw. 34, 1947 (a). - - Auftautiefe und Frostzerrung im Boden Mitteleuropas während der Würmeiszeit. Ein Beitrag zur Bestimmung des Eiszeitklimas. - Naturw. 34, 1947 (b). - - Die Periglazial-Erscheinungen in der Umgebung der Gletscher des Zemmgrundes (Zillertaler Alpen). - Göttinger geogr. Abhandlungen, Heft 15, 1954.

Poser, H. \& Hövermann, J.: Untersuchungen zur pleistozänen Harzvergletscherung. - Abh. braunschw. wiss. Ges. 3, 1951.

SCHENK, E.: Die periglazialen Strukturbodenbildungen als Folgen der Hydratationsvorgänge im Boden. - Eiszeitalter u. Gegenwart 5, 1955. - - Die Mechanik der periglazialen Strukturböden. - Abh. hess. Landesamtes für Bodenforschung, Heft 13, 1955.

Steeger, A.: Diluviale Bodenfrosterscheinungen am Niederrhein. - Geol. Rundschau 34, 1944.

Steusloff, U.: Periglazialer "Tropfen"- und Taschenboden im südlichen Münsterland bei Haltern. - Geol. Jahrb. 66, 1952.

Troll, C.: Strukturböden, Solifluktion und Frostklimate der Erde. - Geol. Rundschau 34, 1944.

VAN Der Vlerk, I. M. \& Florschütz, F.: Nederland in het Ijstijdvak. - Utrecht 1949.

Anschrift des Verf.: Dr. Heinrich Wortmann, Landesgeologe, Krefeld, Cracauerstraße 80. 